\title{
ZERO-ORDER PERTURBATIONS OF THE SUBELLIPTIC LAPLACIAN ON THE HEISENBERG GROUP AND THEIR UNIQUENESS PROPERTIES
}

\author{
NICOLA GAROFALO AND ERMANNO LANCONELLI
}

\section{INTRODUCTION}

The Heisenberg group $\mathbf{H}^{n}$ is the step-two nilpotent Lie group whose underlying manifold is $\mathbf{R}^{2 n+1}$ equipped with the group law $(x, y, t) \circ\left(x^{\prime}, y^{\prime}, t^{\prime}\right)=\left(x+x^{\prime}, y+y^{\prime}, t+t^{\prime}+2\left(x^{\prime} \cdot y-x \cdot y^{\prime}\right)\right)$, where $x \cdot y$ denotes the usual inner product in $\mathbf{R}^{n}$. A basis for the Lie algebra of left-invariant vector fields on $\mathbf{H}^{n}$ is given by

$$
\begin{array}{r}
X_{j}=\frac{\partial}{\partial x_{j}}+2 y_{j} \frac{\partial}{\partial t}, \quad Y_{j}=\frac{\partial}{\partial y_{j}}-2 x_{j} \frac{\partial}{\partial t}, \\
j=1, \ldots, n, \text { and } \frac{\partial}{\partial t} .
\end{array}
$$

It is readily recognized that $\left[X_{j}, Y_{k}\right]=-4 \delta_{j k}(\partial / \partial t)$ so that, in virtue of a fundamental result of Hörmander $[\mathrm{H}]$, the KohnLaplacian

$$
\Delta_{\mathbf{H}^{n}}=\sum_{j=1}^{n}\left(X_{j}^{2}+Y_{j}^{2}\right)
$$

is a second-order hypoelliptic (but not elliptic) operator on $\mathbf{H}^{n}$. In fact, see (7) below, $\Delta_{\mathbf{H}^{n}}$ is real analytic-hypoelliptic, and therefore a solution to $\Delta_{\mathbf{H}^{n}} u=0$ cannot vanish with all its derivatives at one point of a connected open set, unless $u \equiv 0$ in that set.

We are interested in obtaining a quantitative version of the above uniqueness property for solutions to the equation

$$
-\Delta_{\mathbf{H}^{n}} u+V u=0,
$$

Received by the editors November 7, 1989 and, in revised form, December 11, 1989.

1980 Mathematics Subject Classification (1985 Revision). Primary 35B45, 35B60, 35H05, 22E30.

This first author was supported by the NSF, grant DMS-8905338. 
where on the zero-order term $V$ we make suitable assumptions. More explicitly, we seek an estimate of the order of vanishing at one point of a solution $u$ to (3). Such estimate should, in a precise quantitative way, only depend on the local size of $V$ and on suitable $L^{2}$ norms of $u$ and $X_{j} u, Y_{j} u, j=1, \ldots, n$, in some fixed neighborhood of the point in question.

Unfortunately, even when $V$ is $C^{\infty}$, there can be no such estimate for an arbitrary solution $u$ to (3). This is a consequence of a recent negative result of Bahouri [Ba] which is concerned with subelliptic (but not elliptic) operators in $\mathbf{R}^{N}$ of the type $\mathscr{L}=$ $\sum_{j=1}^{N-1} X_{j}^{2}$.

The vector fields $X_{j}$ are requested to satisfy Hörmander's condition on the Lie algebra $[\mathrm{H}]$. Moreover, the linear space generated by them must have dimension $N-1$ at every point. Bahouri proved that if an additional geometric condition is satisfied by the volume form associated to $X_{1}, \ldots, X_{N-1}$, then the operator $\mathscr{L}$ fails to have the unique continuation property in the following sense. There exist a connected open set $\Omega \subset \mathbf{R}^{N}$ and a $V \in C^{\infty}(\Omega)$ such that the equation $-\mathscr{L} u+V u=0$ admits a nontrivial solution in $\Omega$ vanishing in an open subset of $\Omega$. The geometric condition referred to above is trivially satisfied in dimension three or four. As a consequence, when $N=3$ or 4 , every operator of the type described above fails to have the unique continuation property. An interesting example is provided by the operator in $\mathbf{R}^{3}$

$$
\mathscr{L}=\left(\frac{\partial}{\partial x}\right)^{2}+\left(\frac{\partial}{\partial y}-4 x \frac{\partial}{\partial t}\right)^{2}
$$

which satisfies the conditions in [Ba]. Bahouri's construction yields a potential $V \in C^{\infty}(\Omega), \Omega$ a neighborhood of the origin, such that

$$
\operatorname{supp} V \subset\{x \geq 0\}, \quad V \text { is flat at }\{x=0\},
$$

for which there exists a solution to $-\mathscr{L} u+V u=0$ in $\Omega$ supported in $\{x \geq 0\}$. If we consider the change of variables $(x, y, t) \rightarrow$ $\left(x^{\prime}, y^{\prime}, t^{\prime}\right)$ given by $x^{\prime}=x, y^{\prime}=y, t^{\prime}=t-2 x y$, we see that the plane $\{x=0\}$ is mapped into the plane $\left\{x^{\prime}=0\right\}$, and the KohnLaplacian $\Delta_{\mathbf{H}^{1}}=((\partial / \partial x)+2 y(\partial / \partial t))^{2}+((\partial / \partial y)-2 x(\partial / \partial t))^{2}$ is transformed into the operator $\mathscr{L}$ in (4). Therefore, there exists a neighborhood of the origin $\Omega$ in $\mathbf{R}^{3}$ and a $V \in C^{\infty}(\Omega)$ and 
satisfying (5) for which $-\Delta_{\mathbf{H}^{1}}+V$ fails to have the unique continuation property in $\Omega$. When $V$ is real analytic, then a qualitative result of Bony [B] based on Hölmgren's theorem implies the unique continuation property for (3).

In view of the above discussion, is there any positive result when $V$ is not real analytic? We will answer this question affirmatively by providing a sufficient condition for solutions to (3) to have a finite order of vanishing, even when the potential $V$ is allowed quite strong singularities. In order to state our results, we need to introduce some notation.

Henceforth, a generic point $(x, y, t) \in \mathbf{R}^{2 n+1}$ will be denoted by $(z, t)$ whenever convenient. An important group of automorphisms of $\mathbf{H}^{n}$ is given by the so-called Heisenberg dilations $\delta_{\lambda}(z, t)=\left(\lambda z, \lambda^{2} t\right), \lambda>0$. A function $u$ on $\mathbf{H}^{n}$ is said to be Heisenberg-homogeneous of degree $k \in \mathbf{Z}$ if for every $\lambda>0$, $u \circ \delta_{\lambda}=\lambda^{k} u$. There exists a distinguished Heisenberg-homogeneous function of degree one, the distance function (see $[\mathrm{S}]$ and $[\mathrm{F}]$ )

$$
d(z, t)=\left(|z|^{4}+t^{2}\right)^{1 / 4} \text {. }
$$

The homogeneous dimension of $\mathbf{H}^{n}$ (see, e.g., [FS]) is $Q=$ $2 n+2$. It is a remarkable fact that the fundamental solution $\Gamma(z, t)$ of $-\Delta_{\mathbf{H}^{n}}$ with singularity at the origin is

$$
\Gamma(z, t)=\frac{C_{Q}}{d(z, t)^{Q-2}},
$$

where $C_{Q}>0$ is a number depending on $Q$. This was proved by Folland in [F]. Because of (7) the level sets of $\Gamma(z, t)$ are the spheres in the metric generated by (6). Precisely, we let for $r>0$

$$
\begin{gathered}
\boldsymbol{\Omega}_{r}=\left\{(z, t) \in \mathbf{H}^{n} \mid d(z, t)<r\right\}, \\
\partial \boldsymbol{\Omega}_{r}=\left\{(z, t) \in \mathbf{H}^{n} \mid d(z, t)=r\right\},
\end{gathered}
$$

and call these sets, respectively, the Heisenberg ball and sphere centered at the origin with radius $r$.

Let $\Delta_{z}=\sum_{j=1}^{n}\left(\left(\partial^{2} / \partial x_{j}^{2}\right)+\left(\partial^{2} / \partial y_{j}^{2}\right)\right)$ be the Laplacian in the variable $z=(x, y)$ and let $T$ be the vector field

$$
T=\sum_{j=1}^{n}\left(y_{j} \frac{\partial}{\partial x_{j}}-x_{j} \frac{\partial}{\partial y_{j}}\right) .
$$

Then an easy verification yields for $u \in C^{2}\left(\mathbf{H}^{n}\right)$

$$
\Delta_{\mathbf{H}^{n}} u=\Delta_{z} u+4|z|^{2} \frac{\partial^{2} u}{\partial t^{2}}+4 \frac{\partial}{\partial t}(T u) .
$$


Our problem being a local one, we work from now on in a fixed Heisenberg ball $\Omega_{R_{0}}$ centered at the origin. On the zero-order term $V$ in (3) we make the following assumption: There exist $C>0$ and an increasing function $f:\left(0, R_{0}\right) \rightarrow \mathbf{R}^{+}$such that

$$
\int_{0}^{R_{0}} \frac{f(r)}{r} d r<+\infty
$$

and for which

$$
|V(z, t)| \leq C \frac{f(d(z, t))}{d(z, t)^{2}} \psi(z, t) \text { for a.e. }(z, t) \in \Omega_{R_{0}},
$$

where

$$
\psi(z, t)=\frac{|z|^{2}}{d(z, t)^{2}}, \quad(z, t) \neq(0,0) .
$$

The role of the function $\psi$ in (11) will be clarified by Theorems 4 and 5 below. At this moment we simply remark that $0 \leq \psi \leq 1$, $\psi(0, t) \equiv 0, \psi(z, 0) \equiv 1$ and that $\psi$ is Heisenberg-homogeneous of degree zero. Concerning the function $f$ in (11), we note that (10) implies $\lim _{r \rightarrow 0} f(r)=0$. Typical representatives are $f(r)=$ $r^{\varepsilon}, 0<\varepsilon$, and $f(r)=(\log (1 / r))^{-\alpha}, \alpha>1$. According to (11) the zero-order term $V$ in (3) can be quite singular; therefore, a notion of solution to (3) needs to be specified. Since regularity questions are not the main concern for us, we will a priori assume that a solution $u$ to (3) in $\Omega_{R_{0}}$ is a continuous function in $\Omega_{R_{0}}$ such that $u, X_{j} u, Y_{j} u, j=1, \ldots, n$, and $\Delta_{\mathbf{H}^{n}} u$ are in $L^{2}\left(\Omega_{R_{0}}\right)$, and such that $u$ satisfies (3) in the weak sense. We will need the following:

Definition 1. Given a function $u$ such that $\psi^{1 / 2} u \in L^{2}\left(\Omega_{R_{0}}\right)$, we say that $u$ vanishes to infinite order at the origin if as $r \rightarrow 0^{+}$

$$
\int_{\Omega_{r}} u^{2} \psi d z d t=O\left(r^{k}\right), \quad \text { for every } k \in \mathbf{N} \text {. }
$$

Our main result is the following:

Theorem 1. Let $V$ satisfy (11) for some $C$ and $f$. Let $u$ be a solution to (3) in $\Omega_{R_{0}}$. Suppose there exist $C_{1}>0$ and an increasing function $g:\left(0, R_{0}\right) \rightarrow \mathbf{R}^{+}$satisfying (10) and such that

$$
|t T u(z, t)| \leq C_{1} g(d(z, t))|z|^{2}|u(z, t)|, \quad \text { for a.e. }(z, t) \in \Omega_{R_{0}} .
$$


Then there exist two numbers $r_{0}=r_{0}\left(Q, C, C_{1}, f, g\right)>0$ and $\Gamma=\Gamma\left(Q, C, C_{1}, f, g, u\right)>0$ such that, if for every $r \in\left(0, r_{0} / 2\right)$ $u \not \equiv 0$ in $\Omega_{r}$, we have

$$
\int_{\Omega_{2 r}} u^{2} \psi d z d t \leq \Gamma \int_{\Omega_{r}} u^{2} \psi d z d t
$$

Remarks. (a) The constant $\Gamma$ must depend on $u$ as easy examples show. (b) The dependence of both $r_{0}$ and $\Gamma$ on the parameters involved can be made very explicit. In particular, the proof of Theorem 1 yields

$$
\begin{aligned}
\Gamma=2^{Q} \exp \left\{2 \operatorname { l o g } 2 \left[1+\exp \left[M \left(\int_{0}^{R_{0}} \frac{f(r)}{r} d r\right.\right.\right.\right. & \left.\left.\left.+\int_{0}^{R_{0}} \frac{g(r)}{r} d r\right)\right]\right] \\
& \left.\times \max \left(1, N\left(r_{0}\right)\right)\right\},
\end{aligned}
$$

where $M>0$ is a number depending only on $Q, C, C_{1}, f$ and $g$, and

$$
N\left(r_{0}\right)=r_{0} \int_{\Omega_{r_{0}}}\left\{\sum_{j=1}^{n}\left[\left(X_{j} u\right)^{2}+\left(Y_{j} u\right)^{2}\right]+V u^{2}\right\} \frac{d z d t}{\int_{\partial \Omega_{r_{0}}} u^{2} \frac{\psi}{|\nabla d|} d H_{2 n}} .
$$

In the surface integral in the denominator of the above quotient $d H_{2 n}$ stands for $2 n$-dimensional Hausdorff measure in $\mathbf{R}^{2 n+1}$. (c) (13) is trivially satisfied when $T u \equiv 0$. Functions verifying this property can be characterized as those which are invariant with respect to the action of the torus $\mathbf{T}$ on $\mathbf{H}^{n}$ given by $\varphi_{\theta}(\tilde{z}, t)=$ $\left(e^{i \theta} \tilde{z}, t\right), \theta \in[0,2 \pi]$, where we have identified $z=(x, y) \in \mathbf{R}^{2 n}$ with $\tilde{z}=\left(\tilde{z}_{1}, \ldots, \tilde{z}_{n}\right) \in \mathbf{C}^{n}$ with $\tilde{z}_{j}=x_{j}+i y_{j}, j=1, \ldots, n$. We thank David Catlin for pointing out this fact to us. In particular, functions of the type $u(z, t)=u^{*}\left(|z|^{2}, t\right)$ or of the type $u(z, t)=u^{*}\left(\left|\tilde{z}_{1}\right|, \ldots,\left|\tilde{z}_{n}\right|, t\right)$ satisfy $T u \equiv 0$.

Theorem 1 yields the sought quantitative information on the order of vanishing at the origin of a solution to (3) satisfying (13). From it we obtain rather straightforwardly:

Theorem 2. Under the assumptions of Theorem 1, if $u$ vanishes to infinite order at the origin (see Definition 1), then there must be $u \equiv 0$ in $\Omega_{r_{0}}$, where $r_{0}>0$ is as in the conclusion of Theorem 1 .

We emphasize that, given the assumption (11) on $V$, the conclusion of Theorem 1 is simply false if we do not restrict the family of solutions to (3). This can be easily recognized by choosing 
$f(r)=r^{2}$ in (11), so that the latter becomes $|V(z, t)| \leq C \psi(z, t)$, for a.e. $(z, t) \in \Omega_{R_{0}}$.

This requirement is certainly satisfied by the above-mentioned Bahouri's potential in $\mathbf{R}^{3}$, since the latter is flat at $\{x=0\}$; see (5).

\section{SKETCH OF THE PROOF OF THEOREM 1}

Our approach is based on that for elliptic equations found by F. H. Lin and one of us in [GL1] and [GL2]. Our analysis, in fact, shows some remarkable similarities with the euclidean case. Yet, the present subelliptic context offers new and interesting difficulties, some of them of a rather subtle geometrical nature. The main ingredients in the proof of Theorem 1 are:

(I) Representation formulas for smooth functions on $\mathbf{H}^{n}$ as integrals on Heisenberg spheres and balls.

(II) A strong form of uncertainty principle for $\mathbf{H}^{n}$.

(III) First variation estimates for the energy integral associated to $(3)$.

(IV) A frequency function on $\mathbf{H}^{n}$ and the study of its growth properties via parts (I), (II), and (III).

In what follows we briefly outline the implementation of part (IV) in the proof of Theorem 1. At the end of this note we will quote the main results relative to parts (II) and (III), Theorems 4 and 5, respectively. Concerning part (I) we simply mention that our result is an extension to our context of classical formulas involving functions and their Laplacians on $\mathbf{R}^{N}$. It generalizes Gaveau's mean value formula for solutions of $\Delta_{\mathbf{H}^{n}} u=0$; see [G].

By means of Federer's coarea formula [Fe, Theorem 3.2.12], we see that in order to prove (14) it is sufficient to show

$$
\int_{\partial \Omega_{2 r}} u^{2} \frac{\psi}{|\nabla d|} d H_{2 n} \leq \frac{\Gamma}{2} \int_{\partial \Omega_{r}} u^{2} \frac{\psi}{|\nabla d|} d H_{2 n}
$$

for every $r \in\left(0,\left(r_{0} / 2\right)\right)$. To this end we introduce the following height-function in $\partial \Omega_{r}$ of a solution $u$ to (3)

$$
H(r)=\int_{\partial \Omega_{r}} u^{2} \frac{\psi}{|\nabla d|} d H_{2 n}, \quad 0<r<R_{0} .
$$

We also define the following quantities:

$$
D(r)=\int_{\Omega_{r}}\left|\nabla_{\mathbf{H}^{n}} u\right|^{2} d z d t, \quad I(r)=\int_{\Omega_{r}}\left[\left|\nabla_{\mathbf{H}^{n}} u\right|^{2}+V u^{2}\right] d z d t,
$$


which we call, respectively, the Dirichlet integral and the total energy of $u$ in $\Omega_{r}$. In (17) we have let $\left|\nabla_{\mathbf{H}^{n}} u\right|^{2}=\sum_{j=1}^{n}\left[\left(X_{j} u\right)^{2}+\right.$ $\left.\left(Y_{j} u\right)^{2}\right]$. We will adopt this notation from now on.

Lemma 1. Let $u$ be a solution to (3) in $\Omega_{R_{0}}$. Then

$$
H^{\prime}(r)=\frac{Q-1}{r} H(r)+2 I(r) .
$$

The proof of this lemma relies on the representation formulas mentioned in (I) above.

Lemma 2. There exist $r_{0}>0$, depending only on $C, f$ in (11), such that either $u \equiv 0$ in $\Omega_{r_{0}}$ or $H(r) \neq 0$ for every $r \in\left(0, r_{0}\right)$.

The proof of this lemma uses the above-referred uncertainty principle in part (II) (see Theorem 4). Lemma 2 allows to divide out by $H(r)$ in (18) obtaining

$$
\frac{H^{\prime}(r)}{H(r)}=\frac{Q-1}{r}+2 \frac{I(r)}{H(r)}, \quad r \in\left(0, r_{0}\right) .
$$

At this point we introduce the following

Definition 2. The quantity $N(r)=r I(r) / H(r)$ is called the frequency in $\Omega_{r}$ of the solution $u$ to (3).

The motivation for Definition 2 relies in the following:

Proposition 1. Let $u$ be such that $\Delta_{\mathbf{H}^{n}} u=0$ in $\Omega_{R_{0}}$ and $u$ is Heisenberg-homogeneous of degree $k \in \mathbf{N}$. Then $N(r) \equiv k$.

We mention that the frequency of a harmonic function in $\mathbf{R}^{N}$ was first introduced by Almgren [A], who also established its increasingness. Using Definition 2, we rewrite (19) as follows:

$$
\frac{d}{d r}\left[\log \left(\frac{H(r)}{r^{Q-1}}\right)\right]=2 \frac{N(r)}{r}, \quad r \in\left(0, r_{0}\right) .
$$

Following [GL1] and [GL2] we now introduce the set

$$
\Lambda_{r_{0}}=\left\{r \in\left(0, r_{0}\right) \mid N(r)>\max \left(1, N\left(r_{0}\right)\right)\right\} .
$$

Because of Lemma 2 we see that $\Lambda_{r_{0}}$ is an open set of the line. We stress that from the definition of $\Lambda_{r_{0}}$ we have at every $r \in \Lambda_{r_{0}}$

$$
\frac{H(r)}{r}<I(r) \text {. }
$$


The crucial ingredient in the proof of Theorem 1 is the following:

Theorem 3. For a.e. $r \in \Lambda_{r_{0}}$ we have

$$
\frac{N^{\prime}(r)}{N(r)} \geq-M\left(\frac{f(r)}{r}+\frac{g(r)}{r}\right)
$$

where $M>0$ is a constant which solely depends on $Q, C, C_{1}$, and $f$ and $g$.

The proof of Theorem 3 is quite delicate and is based on parts (II) and (III) above, along with, of course, use of assumptions (11) and (13). With (21) in hand it is not difficult, upon integrating (20) over an interval $(r, 2 r)$, to deduce (15) with a constant $\Gamma$ as in (b) of the remarks above. This would finish the proof of Theorem 1.

To conclude we would like to say something about (II) and (III).

(II). A relevant role in [GL2] was played by the following strong form of Heisenberg's uncertainty principle

$$
\begin{aligned}
& \int_{|x|<r} \frac{u^{2}}{|x|^{2}} d x \\
& \quad \leq\left(\frac{2}{N-2}\right)^{2}\left\{\left(\frac{N-2}{2}\right) \frac{1}{r} \int_{|x|=r} u^{2} d x+\int_{|x|<r}|\nabla u|^{2} d x\right\},
\end{aligned}
$$

valid for any function $u \in C_{0}^{\infty}\left(\mathbf{R}^{N} \backslash\{0\}\right)$. This implies by Schwarz's inequality

$$
\left(\int_{\mathbf{R}^{n}}|x|^{2} u^{2} d x\right)\left(\int_{\mathbf{R}^{N}}|\nabla u|^{2} d x\right) \geq\left(\frac{N-2}{2}\right)^{2}\left(\int_{\mathbf{R}^{N}} u^{2} d x\right)^{2},
$$

which via Plancherel's theorem for the Fourier transform yields the harmonic analysis formulation of Heisenberg's uncertainty principle in $\mathbf{R}^{N}$; see [He, Chapter 2]. (With $(N-2) / 2$ replaced by $N / 2$ equality is attained iff $u(x)=A \exp \left(-\alpha|x|^{2}\right)$, for some $A \in \mathbf{R}$, $\alpha>0$.)

In our context we prove:

Theorem 4. For every $u \in C_{0}^{\infty}\left(\mathbf{H}^{n} \backslash\{(0,0)\}\right)$ and every $r>0$, we 
have

$$
\begin{aligned}
& \int_{\Omega_{r}} \frac{u^{2}}{d^{2}} \psi d z d t \\
& \leq\left(\frac{2}{Q-2}\right)^{2}\left\{\left(\frac{2}{Q-2}\right)^{2} \frac{1}{r} \int_{\partial \Omega_{r}} u^{2} \frac{\psi}{|\nabla d|} d H_{2 n}\right. \\
&\left.\quad+\int_{\Omega_{r}}\left|\nabla_{\mathbf{H}^{n}} u\right|^{2} d z d t\right\} .
\end{aligned}
$$

Corollary 1 (Uncertainty principle for $\mathbf{H}^{n}$ ). For every $u$ such that $\psi^{1 / 2} u, X_{j} u, Y_{j} u \in L^{2}\left(\mathbf{H}^{n}\right), j=1, \ldots, n$, we have

$$
\begin{gathered}
\left(\int_{\mathbf{H}^{n}} d^{2} u^{2} \psi d z d t\right)\left(\int_{\mathbf{H}^{n}}\left|\nabla_{\mathbf{H}^{n}} u\right|^{2} d z d t\right) \\
\geq\left(\frac{2}{Q-2}\right)^{2}\left(\int_{\mathbf{H}^{n}} u^{2} \psi d z d t\right)^{2} .
\end{gathered}
$$

One can prove that with $(Q-2) / 2$ replaced by $Q / 2$ equality is attained in Corollary 1 iff $\left.u(z, t)=A \exp (-\alpha d) z, t)^{2}\right)$, for some $A \in \mathbf{R}, \alpha>0$.

(III). First variation estimates have an important role in calculus of variations and geometric measure theory. The general strategy to obtain them is to perform a so-called radial deformation and then use the minimizing property of the energy integral. In euclidean flat space the effectiveness of this procedure is deeply related to the fact that the (conformal) vector field $X=r(r / \partial r)$ is orthogonal to the level sets of the fundamental solution of Laplace's operator. This is best illustrated by the first variation formula for the Euclidean Dirichlet integral of a function

$$
\begin{aligned}
\int_{\partial B_{r}}|\nabla u|^{2} d \sigma= & \frac{N-2}{r} \int_{B_{r}}|\nabla u|^{2} d x \\
& +2 \int_{\partial B_{r}}\left(\frac{\partial u}{\partial n}\right)^{2} d \sigma-\frac{2}{r} \int_{B_{r}}(x \cdot \nabla u) \Delta u d x,
\end{aligned}
$$

where we have set $B_{r}=\left\{x \in \mathbf{R}^{N}|| x \mid<r\right\}$ and $(\partial u / \partial n)=$ $\nabla u \cdot(x /|x|)$. It is noticeable in this formula the absence of terms containing tangential derivatives of $u$.

On the Heisenberg group the situation is quite different. Yet, there exists a distinguished vector field which plays to some extent the same fundamental role played by the vector field $r(\partial / \partial r)$ in 
$\mathbf{R}^{N}$. Namely,

$$
X=\sum_{j=1}^{n}\left(x_{j} \frac{\partial}{\partial x_{j}}+y_{j} \frac{\partial}{\partial y_{j}}\right)+2 t \frac{\partial}{\partial t} .
$$

A (somewhat magic) redeeming feature of this vector field $X$ is that its projection along the direction orthogonal to a level set of $\Gamma$ in (7), i.e., the Heisenberg sphere, is constant along the sphere itself. We exploit this fact to obtain the following remarkable sub-elliptic first variation formula.

Theorem 5. Let $u$ be a function such that $u, X_{j} u, Y_{j} u, j=$ $1, \ldots, n$, and $\Delta_{\mathbf{H}^{n}} u \in L^{2}\left(\mathbf{H}^{n}\right)$. Then we have for a.e. $r>0$

$$
\begin{aligned}
D^{\prime}(r)= & \frac{Q-2}{r} D(r)+2 \int_{\partial \Omega_{r}}\left(\frac{X u}{r}\right)^{2} \frac{\psi}{|\nabla d|} d H_{2 n} \\
& +2 \int_{\partial \Omega_{r}}\left(\frac{X u}{r}\right)\left(\frac{T u}{r}\right) \frac{\varphi}{|\nabla d|} d H_{2 n} \\
& -\frac{2}{r} \int_{\Omega_{r}}(X u)\left(\Delta_{\mathbf{H}^{n}} u\right) d z d t .
\end{aligned}
$$

In (23) $D(r)$ is defined by (17), $X$ by (2), $T$ by (8), and in the third integral in the r.h.s. we have let $\varphi=\varphi(z, t)=t /\left(d(z, t)^{2}\right)$. (Notice that $\varphi$ is Heisenberg-homogeneous of degree zero.) One should compare (23) with the above formula for the euclidean Dirichlet integral, and notice the presence in (23) of the boundary integral containing the term $T u$. Since it can be easily shown that the vector field $T$ in (8) is tangential to the Heisenberg sphere, the third term in the r.h.s. of (23) represents a novelty w.r.t. the euclidean case, and a bad one, indeed. Theorem 5 is the main tool in the proof of Theorem 3. We obtain it as a consequence of a general integral identity of Rellich type for $\mathbf{H}^{n}$. We believe this identity will prove useful for other problems on the Heisenberg group, as well.

A conclusive remark. Condition (13) is only sufficient for the estimate (14) to hold. By (20) and Proposition 1 every Heisenberghomogeneous solution of $\Delta_{\mathbf{H}^{n}} u=0$ satisfies (14). Yet, not every such function satisfies (13) (e.g., $u(x, y, t)=x|z|^{2}+2 t y$ ). An interesting open question, which we have not been able to settle yet, is to characterize the class of solutions to (3) which satisfy (14). 


\section{REFERENCES}

[A] F. T. Almgren, Jr., Dirichlet's problem for multiple valued functions and the regularity of mass minimizing integral currents, Minimal Submanifolds and Geodesics (M. Obata, ed.), North-Holland, Amsterdam, 1979, pp. 1-6.

[Ba] H. Bahouri, Non prolongement unique des solutions d'operateurs "Somme de Carrés", Ann. Inst. Fourier (Grenoble) 36 (4) (1986), 137-155.

[B] J. M. Bony, Principe du maximum, inéqalité de Harnack et unicité du problème de Cauchy pour les opérateurs elliptiques dégénérés, Ann. Inst. Fourier (Grenoble) 19 (1) (1969), 277-304.

[Fe] H. Federer, Geometric measure theory, Grundlehren Math. Wiss., vol. 153, Springer-Verlag, Berlin, 1969.

[F] G. B. Folland, A fundamental solution for a subelliptic operator, Bull. Amer. Math. Soc. 79 (2) (1973), 373-376.

[FS] G. B. Folland and E. M. Stein, Hardy spaces on homogeneous groups, Math. Notes, Princeton Univ. Press, Princeton, N. J., 1982.

[GL1] N. Garofalo and F. H. Lin, Monotonicity properties of variational integrals, $A_{p}$ weights and unique continuation, Indiana Univ. Math. J. 35 (2) (1986), 245-268.

[GL2] _- Unique continuation for elliptic operators: A geometric-variational approach, Comm. Pure Appl. Math. XL (1987), 347-366.

[G] B. Gaveau, Principe de moindre actions, propagation de la chaleur et estimées sous elliptiques sur certains groups nilpotents, Acta Math. 139 (1977), 95-153.

[He] W. Heisenberg, The physical principles of the quantum theory, Dover, 1949.

[S] E. M. Stein, Some problems in harmonic analysis suggested by symmetric spaces and semi-simple groups, Actes, Congrés intern. math., Nice, 1 (1970), 173-189.

Department of Mathematics, Purdue University, West lafayette, INDIANA 47907

Dipartimento di Matematica, Universitá di Bologna, Piazza di Porta S. Donato 5, 40127 Bologna, Italy 
